\title{
Competencias técnicas de los trabajadores informáticos El caso de Argentina
}

\author{
José Borello, Analía Erbes, \\ Verónica Robert, Sonia Roitter y Gabriel Yoguel
}

José Borello

Investigador-docente,

Instituto del Conurbano, Universidad

Nacional de General Sarmiento (UNGS)

œ jborello@ungs.edu.ar

Analía Erbes

Investigadora-docente

Instituto de Industria, UNGS

œ aerbes@ungs.edu.ar

Verónica Robert

Investigadora-docente,

Instituto de Industria, UNGS

• vrobert@gmail.com

Sonia Roitter

Investigadora-docente,

Instituto de Industria, UNGS

• sroitter@ungs.edu.ar

Gabriel Yoguel

Investigador-docente

Instituto de Industria, UNGS

• gyoguel@ungs.edu.ar

ste artículo presenta un balance de las competencias de los recursos humanos informáticos de Argentina. En diversos espacios gubernamentales, empresariales y académicos argentinos se da por sentada la existencia de bondades y potencialidades de los recursos humanos locales en esta disciplina. Tal creencia es abonada por una historia de la informática nacional que es rica aunque contradictoria, pero que no se apoya en análisis de la calidad de esos recursos humanos. El presente estudio apunta a desarrollar y aplicar una metodología que permita evaluar las competencias de los trabajadores informáticos, señalando sus problemas y posibilidades, sobre la base de los resultados de una encuesta electrónica. Los rasgos actuales de esos recursos humanos y su heterogeneidad son interpretados a partir de los avances y retrocesos de la actividad a lo largo de su sendero evolutivo. 


\section{I}

\section{Introducción}

En las últimas décadas el sector informático argentino ha sido considerado más de una vez como un elemento clave para la modernización del país. Esto ha permitido que Argentina tenga ya cierta trayectoria en la informática tanto en la academia como, en menor medida, en la actividad empresarial. Podría sugerirse que los rasgos idiosincrásicos del sector y la heterogeneidad actual de sus recursos humanos se derivan de los avances y retrocesos que se dieron a lo largo de su sendero evolutivo. A partir de la crisis del modelo de convertibilidad (diciembre del 2001), resurgió un fuerte interés en el desarrollo de este sector, intensivo en conocimiento, bajo el supuesto de la existencia de una masa crítica de recursos humanos de alta calidad.

Sin embargo, las evidencias empíricas cuestionan parcialmente la existencia de capacidades suficientes para contribuir a una expansión del sector informático en el país. Gran parte de los estudios sobre el uso y la producción de tecnologías de la información y de la comunicación (TIC) realizados en Argentina en los últimos años, ha señalado las limitaciones existentes para difundir y desarrollar una industria competitiva de software y servicios informáticos. Los trabajos que se centran en los problemas de difusión de las TIC muestran que esos problemas tienen relación directa con el tamaño de las empresas, el nivel alcanzado por las competencias endógenas de los agentes y la presencia de redes de empresas (Novick, 2002; Boscherini, Novick y Yoguel, 2003; Martin y Rotondo, 2005; Yoguel, Novick y otros, 2004; Cabello y Moyano, 2005; Lugones, Bianco y otros, 2003). Estos estudios también muestran que en las empresas se hace mayor uso de las TIC en las áreas administrativas, lo que implica una escasa difusión de herramientas complejas, un uso poco sistemático de ellas y pocas transformaciones derivadas de la incorporación de las TIC en la organización de la producción.

Desde la perspectiva de la oferta, los estudios realizados han puesto de relieve que en el sector informático del país no existe una masa crítica de empresas de excelencia y que en él predominan las activida-

Los autores agradecen los comentarios de Alice Lam, BengtAke Lundval, Franco Malerba y Gabriel Baum a versiones anteriores de este trabajo. des de servicios más que el desarrollo de productos (López, 2003; Chudnovsky, López y Melitzko, 2001; Perazzo, Delbue y otros, 1999). Asimismo, los sectores más dinámicos de la economía (los complejos agroalimentarios y energéticos) no generan una demanda sobre el sector que pueda impulsar un aprendizaje importante e incrementar la oferta exportable de software.

La síntesis que hemos hecho de la bibliografía existente muestra los problemas de oferta y demanda y pone de relieve que una visión transversal centrada en los recursos humanos ayudaría a entender parte de esas limitaciones. Por lo tanto, contar con un balance de las competencias técnicas de los recursos humanos informáticos permitiría disponer de más elementos para analizar sus potencialidades.

El presente artículo apunta a construir y aplicar una metodología que permita evaluar las competencias de los trabajadores informáticos argentinos, a partir de los resultados de una encuesta electrónica. ${ }^{1}$ Sobre la base de esta encuesta, pretende brindar elementos para evaluar las posibilidades de que, en los próximos años, Argentina pueda elevar significativamente su capacidad de resolver problemas complejos en el sector informático, vinculados tanto al mercado interno como al internacional.

Las publicaciones recientes sobre organización empresarial y desarrollo económico vienen señalando que, en el estudio de las actividades ligadas a las nuevas tecnologías, el enfoque que se centra en los recursos humanos complementa el análisis convencional centrado en las empresas (Markusen, 2002). Se argumenta, por una parte, que se puede identificar un conjunto de nuevas formas empresariales que surgen de la interacción de empresas e instituciones y no dentro de ellas y, por otra, que los trabajadores tienden a llevar a cabo tareas en diversos lugares al mismo tiempo o a cambiar frecuentemente de empresa o institución (Micheli, 2003). Así, ya no existe una identificación clara de los trabajadores con una determinada empresa o institución, sino con proyectos o con comunidades epistémicas transversales a los límites legales de

\footnotetext{
${ }^{1}$ La encuesta fue realizada conjuntamente por la Sociedad Argentina de Informática e Investigación Operativa y la Universidad Nacional de General Sarmiento (encuesta SADIO-UNGS).
} 
las organizaciones (Nemirovsky y Yoguel, 2001). En actividades que se sustentan en la información y el conocimiento son relevantes las "comunidades y las redes de prácticas" (Brown y Duguid, 2000) y las "comunidades de conocimiento" (Henry y Pinch, 2000): ellas son los vehículos que permiten trascender los límites formales de las empresas e instituciones. Las competencias de los trabajadores son clave para la creación de ventajas competitivas dinámicas, para la construcción de redes y la circulación de conocimiento, y para explicar la innovación al interior de las empresas (Metcalfe, Ramlogan y Uyarra, 2002). La mayoría de los estudios existentes concuerdan en señalar que, por las mismas características de las actividades que se realizan, los recursos humanos son un prisma privilegiado de comprensión y análisis del sector (Ducatel, 1994).

Para evaluar las capacidades de los recursos humanos en el sector informático es preciso buscar respuesta a varias interrogantes, entre las cuales se pueden mencionar las siguientes: ¿Cuáles son los diferentes perfiles de los trabajadores informáticos y en qué medida esos perfiles se derivan de la evolución del sector? ¿Cuentan estos trabajadores con las capacidades nece- sarias para desarrollar una industria local competitiva de software y servicios informáticos que pueda enfrentar los desafíos de los sectores más dinámicos, tanto en el mercado interno como en el externo? ¿Contribuye la estructura económica local a dinamizar el sector? ¿Se identifican los trabajadores informáticos con algún proyecto del que forman parte, o llevan adelante actividades individuales? ¿El sistema educativo está formando recursos humanos capaces de abordar un software complejo y de adaptarse exitosamente a los cambios tecnológicos en curso? ¿Cuál es la relación existente entre la generación de competencias técnicas y la participación en redes e instituciones? Nuestro artículo pretende responder algunas de estas preguntas.

Tras la presente sección introductoria, la sección II sintetiza ciertos elementos estructurales del sector informático y describe en forma estilizada su sendero evolutivo en las últimas décadas, desde la perspectiva de la formación de capacidades. La sección III presenta el marco conceptual y metodológico y la hipótesis del trabajo. La sección IV examina la información y pone a prueba la hipótesis mencionada, a través de un análisis de grupos (clusters). En la sección V, por último, se plantean las principales conclusiones.

\section{II}

\section{Estructura y evolución de la informática en Argentina}

\section{Rasgos estructurales y diferencias con los países de inserción tardía exitosa}

Entre los denominados países de ingreso tardío al mercado internacional de informática, Argentina ocupa una posición relativamente marginal (Anlló, Bezchinsky y otros, 2003). Su sector informático es muy pequeño en comparación con los de países como India, Irlanda e Israel, que son los casos de evolución exitosa de los últimos diez años (Arora y Gambardella, 2005). Así, por ejemplo, las ventas de Irlanda e India son, respectivamente, 33 y 30 veces superiores a las de Argentina, y las de Israel 10 veces superiores. Desde la perspectiva del empleo, las diferencias son de 16 veces en el caso de India, pero menos marcadas respecto de los otros dos países: Irlanda tiene el doble de ocupados e Israel un número de ocupados relativamen- te similar al de Argentina (alrededor de 15.000 personas). Esto se manifiesta en fuertes diferencias en las ventas por ocupado entre los países mencionados. Mientras que en India el indicador dobla al de Argentina, en Israel es 10 veces superior y en Irlanda 17 veces mayor. En este último caso, la diferencia se debe básicamente a las ventas por ocupado de las empresas transnacionales (cifra 28 veces superior) y en menor medida de los agentes locales (cifra cuatro veces superior) ${ }^{2}$

\footnotetext{
${ }^{2}$ Los elevados valores que exhibe el indicador de ventas por ocupado en Irlanda e Israel se deben fundamentalmente a que en las tareas informáticas que realizan estos países se hace uso menos intensivo de fuerza de trabajo. Esto se debe en gran parte a la importancia de la subcontratación externa en países como Irlanda donde las labores menos complejas y con uso más intensivo de personal - por ejemplo, las de codificación y de verificación- se
} 
Hay otras diferencias significativas en la inserción externa: Argentina exhibe un coeficiente de exportación significativamente más bajo (17\%) que el de los otros tres países mencionados (75\%). A la vez, la importancia del sector informático en la economía argentina es significativamente menor.

Respecto a otros países latinoamericanos, las diferencias entre sectores informáticos también son importantes. Las ventas y el nivel de empleo de Brasil son, respectivamente, ocho y nueve veces más altos que los de Argentina, aunque están orientados fundamentalmente al mercado interno. Chile y Uruguay tienen sectores informáticos relativamente más pequeños en términos de ventas. El de Uruguay se destaca por su elevado coeficiente de exportación (33\%).

Sin embargo, diversos autores (entre ellos López, 2003; Anlló, Bezchinsky y otros, 2003) coinciden en que el avance de la informática en Argentina ha sido importante, al menos en términos cuantitativos. Esta evolución pudo estar sustentada en la historia del sector, como veremos más adelante, así como en la creación de capacidades en los recursos humanos. A pesar de los esfuerzos realizados en el sistema educativo, hay importantes falencias que se vinculan en parte a marchas y contramarchas en programas que habían sido considerados estratégicos y al reciente surgimiento anárquico de diversas carreras e institutos de formación en informática ${ }^{3}$ - de carácter técnico, profesional y de posgrado - producto del fuerte crecimiento del sector. Este crecimiento vino acompañado de una gran diversidad de carreras, contenidos, creación de competencias y cargas horarias (Aguirre, 2003; Monteverde y Pérez, 2005), lo que ha contribuido a que exista mucha incertidumbre respecto a las capacidades de los graduados en los diversos niveles de formación.

El primer nivel —educación técnica profesional a nivel medio - tiene como objetivo generar capacidades para la programación, el diseño y la codificación. Actualmente representa un tercio de los casi 60.000 estudiantes de informática en el país. El segundo nivel —educación técnica superior no universitariaapunta a formar analistas de sistemas que diseñen,

transfieren a países como India, donde la hora/programador es más barata. En este sentido, el indicador de ventas por ocupado no puede equipararse al de productividad, por el peso de las importaciones (especialmente en el caso de Irlanda, donde la presencia de empresas transnacionales es crucial).

${ }^{3}$ Por ejemplo, entre 1997 y 2002 los egresados de carreras de informática de grado aumentaron $151 \%$ (de 1.000 a 2.627). Tras constituir el 12\% de los egresados de ciencias aplicadas en 1997 pasaron a ser el $20 \%$ en 2002 . desarrollen, integren y verifiquen sistemas de software; atrae a más de la mitad de los alumnos e involucra a unas 30.000 personas en cursos de aproximadamente tres años. El tercer nivel —universitario de gradoforma ingenieros en software y licenciados en sistemas y en informática, capaces de establecer estándares tecnológicos y metodológicos, de construir herramientas que enriquezcan el ambiente de desarrollo, y de gestionar procesos de software. En este grupo se halla poco más del 10\% (7.000) de los estudiantes de informática de todos los niveles. Por último, el nivel de posgrado, de reciente creación en Argentina, incluye alrededor de 37 programas y presumiblemente abarca menos del $1 \%$ del total de estudiantes de informática. ${ }^{4}$

En los tres primeros niveles se observa una gran diversidad de títulos (carreras), de contenidos y de horas/cátedra, incluso bajo iguales denominaciones. ${ }^{5}$ Asimismo, es dudoso que se disponga de suficientes profesores idóneos para proveer educación de calidad en todas las instituciones existentes. Por otra parte, pese al fuerte crecimiento de estas instituciones, sigue siendo importante el número de personas autodidactas en informática o formadas en el ámbito laboral. ${ }^{6}$ Es difícil, por lo tanto, conocer los alcances y competencias de los recursos humanos existentes.

A esto se agrega que solo existen evaluaciones de calidad para algunas carreras de grado y estudios de posgrado, y hay casos en que los programas de formación universitaria se equiparan a los de educación terciaria no universitaria. ${ }^{7}$ Esto pone de manifiesto que existe mucho desconocimiento de la calidad de la formación media y terciaria no universitaria, y una gran heterogeneidad de estudios de grado y de posgrado.

La falencia más importante de un número significativo de carreras universitarias está vinculada con la falta de investigación que acompañe a la actividad docente, lo que en algunos casos se traduce en baja

\footnotetext{
${ }^{4}$ Véase información más desagregada en el informe anual de la Dirección Nacional de Información y Evaluación de la Calidad Educativa (DINIECE), en informes de la Secretaría de Política Universitaria y en Anlló, Bezchinsky y otros (2003).

${ }^{5}$ Algunos de los títulos son, entre otros, los de analista de sistemas, técnico informático, ingeniero de software, ingeniero de sistemas, licenciado en sistemas, licenciado en informática y licenciado en ciencias de la computación.

${ }^{6}$ Situación que se pone de manifiesto en los resultados de la encuesta SADIO-UNGS.

${ }^{7}$ Algunos informes de la Comisión Nacional de Evaluación y Acreditación Universitaria (CONEAU) contienen recomendaciones como la siguiente: se "deberá realizar un gran esfuerzo de transformación curricular y de perfil del cuerpo docente para diferenciarla de la formación que brindan muchos institutos terciarios" (www.coneau.gov.ar).
} 
productividad de las áreas de investigación y, en otros, simplemente en su inexistencia. Esa falta de investigación impide marcar diferencias entre las carreras universitarias y los institutos de formación terciaria no universitaria, y podría estar produciendo una devaluación de los títulos universitarios. Por otra parte, las áreas de investigación de las universidades nacionales muchas veces están más vinculadas a esfuerzos de personas que a políticas institucionales de largo plazo, por lo que sus actividades son altamente inestables $\mathrm{y}$ atomizadas.

\section{Evolución histórica del sector informático}

En Argentina este sector tiene una larga tradición que se inició hacia fines de la década de 1950 y que no puede entenderse disociada del desarrollo de la ciencia y la tecnología en el país, particularmente en el período de industrialización mediante la sustitución de las importaciones (ISI). Este modelo de desarrollo determinó un patrón de especialización que fue acompañado por un conjunto de instituciones, las cuales contribuyeron a que hubiera avances tecnológicos locales. La evolución de la informática, sin embargo, no fue lineal, sino que registró avances y retrocesos (Azpiazu, Basualdo y Nochteff, 1990; Babini, 2003).

Los inicios del desarrollo local del sector pueden remontarse a los últimos años del decenio de 1950, con continuidad en los de 1960 y 1970. En la primera mitad de la década de 1960 el sector informático argentino llegó a ser líder en América Latina. Se trataba de una actividad incipiente en un área igualmente incipiente a nivel mundial, y su avance estaba mucho más vinculado al de los equipos que lo que estaría unas décadas después. En los decenios de 1970 y 1980 hubo progresos significativos ligados tanto a inversión extranjera directa como a empresas nacionales. El crecimiento alcanzado por estas últimas fue particularmente notable para la época, en el marco de un programa de sustitución de importaciones y acuerdos de transferencia tecnológica con empresas trasnacionales líderes en el sector.

Si bien no puede hablarse de un punto de quiebre exacto del modelo sustitutivo de importaciones, la interrupción de la democracia en 1976 sí constituyó una fecha clave. Aquel esquema de desarrollo fue desarticulándose paulatinamente, tanto desde la perspectiva del patrón de especialización como de las instituciones y regulaciones que le eran funcionales. Así, se transitó hacia una especialización fuertemente basada en el aprovechamiento de ventajas comparati- vas estáticas y hacia la desregulación de los mercados y una menor participación del Estado en la esfera económica.

El intento aperturista de fines de la década de 1970, centrado en el enfoque monetario del balance de pagos, no incluía necesariamente una menor participación del Estado en la producción de bienes y servicios, pero sí una ruptura drástica respecto de las instituciones más vinculadas a la producción de conocimiento. Luego de la crisis de la deuda, y con el retorno de la democracia, en 1983, hubo un intento de recuperar algunas de las instituciones científicas y técnicas. Sin embargo, la falta de esfuerzos significativos por reemplazar la especialización basada en recursos naturales y productos intermedios (commodities) por un esquema de ventajas comparativas estáticas contribuyó a crear una brecha considerable entre las capacidades y conocimientos construidos en universidades e institutos de investigación y las necesidades del sistema productivo.

En la década de 1980 se formularon por primera vez lineamientos de una política para el sector informático, que se aproximaban a las propuestas que podían encontrarse en países desarrollados o de industrialización acelerada. Dichos lineamientos, orientados al desarrollo del complejo electrónico en general y al segmento de la informática en particular, se originaron en material gestado por la Comisión Nacional de Informática creada en 1984. Entre las metas e instrumentos se contemplaban desgravaciones impositivas basadas en concursos públicos, preferencias para las empresas de capital nacional y políticas de "compre nacional" en productos de software. También se preveían acciones que apuntaban a generar encadenamientos de creciente complejidad, vincular la oferta local de software con la demanda, formar recursos humanos especializados, negociar acuerdos con países latinoamericanos y promover la creación de software en español (Aspiazu, Basualdo y Nochteff, 1990).

Dentro de ese marco se estableció en 1986 el Programa Argentino Brasileño de Investigación y Estudios Avanzados en Informática (PABI), mediante un convenio entre ambos países encaminado a llevar a cabo proyectos conjuntos, intercambiar recursos humanos y realizar encuentros. El comienzo de este programa puso de manifiesto que, debido a la carencia de grupos consolidados en Argentina, la primera etapa debía dedicarse a la formación de recursos humanos. Esto desembocó en la fundación de las Escuelas Argentino Brasileñas de Informática (EBAI), para contribuir a formar una masa crítica de investigadores, a calificar la 
enseñanza de grado y a crear una escuela de pensamiento regional en la disciplina ${ }^{8}$ (Aguirre, 2003).

Como parte de este proceso de intercambio con Brasil y para mejorar significativamente la formación de recursos humanos, en 1986 se creó la Escuela Superior Latinoamericana de Informática (ESLAI). Aunque esta escuela preparaba recursos humanos de excelencia, no hacía hincapié en la creación de nexos con el mundo empresarial que permitieran tanto iniciar nuevas actividades como efectuar trabajos vinculados con la industria y el sector público (Aguirre, 2003). Esta falencia debida al predominio del modelo lineal de innovación, sumada a un marco macroeconómico adverso, limitaron sus posibilidades de afianzarse en la sociedad y su continuidad misma. No obstante, la ESLAI contribuyó en parte a contrarrestar las deficiencias de calidad y cantidad de recursos humanos dedicados a la informática: muchas de las personas que se incorporaban al sector provenían de una generación anterior formada en el contexto del modelo de industrialización mediante la sustitución de importaciones (Aspiazu, Basualdo y Nochteff, 1990).

Las políticas diseñadas en este período se diferenciaron tanto de las implementadas durante la ISI como de aquellas asociadas a la liberalización económica de la década de 1970, por lo que constituyeron un aporte novedoso y único en la historia del sector. Sin embargo, no tuvieron continuidad debido a: i) la ausencia de una visión global de desarrollo que contemplara un perfil de especialización más complejo y la construcción de nexos entre la academia y el sistema productivo, ii) la inestabilidad macroeconómica y iii) el proceso de reestructuración regresiva del sector manufacturero y del Estado.

Durante el decenio de 1990 se intensificó la apertura comercial y financiera y la desregulación de los mercados, se redujo la participación del Estado en la economía y se profundizó el perfil de especialización. En este período predominó una visión de tecnología según la cual el conocimiento incorporado en bienes de capital importados se difundiría en la estructura productiva y en la sociedad, constituyendo un elemento clave para el incremento de la productividad. El significativo aumento de las importaciones de software y

\footnotetext{
${ }^{8}$ Entre 1986 y 1989 se realizaron cuatro experiencias en Argentina y Brasil, interrumpiéndose este proceso de formación de recursos humanos a fines del decenio de 1980. Como resultado recibieron formación 2000 estudiantes y se publicaron sesenta títulos originales en portugués y español, que aún se usaban a mediados de la década de 1990 en las carreras de informática.
}

equipos informáticos durante este período relegó a los trabajadores del sector fundamentalmente a tareas de adaptación y adecuación de productos importados. A la vez, la existencia de estándares cerrados y plataformas propietarias de los productos importados restringió las posibilidades de aprendizaje y profundizó la dependencia tecnológica. Por otra parte, contrarrestando estos efectos, la modernización de los servicios a partir del proceso de privatización indujo una fuerte demanda de nuevos productos de software que en parte fue satisfecha por empresas locales.

Por otro lado, en la primera mitad del decenio de 1990 se desmontaron los instrumentos de política concebidos en la década anterior, marcando un quiebre en la continuidad institucional. Sin embargo, a partir de la segunda mitad de dicho decenio hubo esfuerzos e inversiones en el sistema científico y tecnológico que llevaron a la creación de universidades y de diversos programas para reforzar las actividades de formación e investigación. Como ejemplos cabe mencionar la fundación de la Agencia Nacional de Promoción Científica y Tecnológica, que administra los fondos obtenibles por concurso para proyectos científicos y tecnológicos, y el Fondo para la Mejora de la Calidad en la Enseñanza de grado de las Ciencias (FOMEC), programa orientado a fortalecer las capacidades de formación universitaria. A mediados del decenio, la evaluación inicial de este programa confirmó el diagnóstico efectuado por el PABI en la segunda mitad de la década de 1980 sobre las debilidades de los recursos humanos en el sector informático. ${ }^{9}$ De los 202 millones de dólares otorgados por el FOMEC, el 5\% asignado a proyectos de informática permitió mejorar el equipamiento, dotar bibliotecas y, sobre todo, realizar una importante actividad de formación de recursos humanos a nivel de doctorado. ${ }^{10}$

Si bien este programa, que finalizó en 1999, contribuyó a la formación de recursos humanos, se dio en el marco de una fuerte desvinculación entre ciencia, tecnología y sistema productivo, y de consolidación de

\footnotetext{
${ }^{9}$ Las 21 universidades nacionales con carreras de informática tenían casi 5000 alumnos con una tasa de egreso de sólo 3\% de los que ingresaban en el mismo período. A su vez, la planta docente global incluía solo dos doctores y la disponibilidad de equipos era ínfima, llegando en algunos casos a un puesto equipado cada 50 alumnos. Lo mismo sucedía con las bibliotecas, que disponían de 0,65 libros por alumno. Solo tres universidades habían iniciado recientemente carreras de doctorado con dirección externa.

${ }^{10}$ Casi $30 \%$ de los fondos otorgados para informática se destinó a doctorados, lo que contribuyó a que a principios de la década del 2000 el número de doctores pasara a 70.
} 
un perfil de especialización productiva que significaba para el sector una demanda de escasa complejidad. Por lo tanto, a pesar de esos esfuerzos, las diferencias entre las necesidades del sistema de producción y las capacidades generadas por el sistema educativo se pusieron nuevamente de manifiesto. ${ }^{11}$ En consecuencia, el perfil de recursos humanos que el sistema educativo fue generando en el área de ciencia y tecnología estuvo más relacionado con la estructura sectorial de importaciones que con la estructura y especialización productivas locales (Nemirovsky y Yoguel, 2004). Esta contradicción, recurrente en los distintos períodos, se manifestó en fugas de cerebros ${ }^{12}$ (Albornoz, Luchilo y otros, 2003; Suárez, 2004).

La crisis reciente del modelo económico predominante en la década de 1990, que se desencadenó luego de la devaluación de 2002, ha reabierto el debate sobre la necesidad de introducir elementos más complejos en el patrón de especialización del país. La inclusión de actividades que hacen uso intensivo de la información y el conocimiento, que se asocian a rendimientos crecientes a escala y a la creación de redes, y que exhiben elevada elasticidad-ingreso de la demanda y fuerte dinamismo en el mercado internacional, permitiría aprovechar ventajas comparativas dinámicas y aminorar la vulnerabilidad macroeconómica (Reinert, 2002; Lall, 2001; Guerrieri, 1993; Dosi, Pavitt y Soete, 1990).

En ese marco, ha aumentado el interés de los agentes económicos y del gobierno en el desarrollo del sector informático, visto como elemento clave para la modernización del país. En particular, estos protagonistas aprecian que el sector tiene grandes potencialidades que residen sobre todo en las capacidades de sus recursos humanos y en una historia previa que ha contribuido a generar esas competencias.

\section{III}

\section{Hipótesis del trabajo y modelo de análisis}

Teniendo como precedente el contexto histórico examinado y la gravitación de las competencias de los trabajadores informáticos en el desempeño del sector, en esta sección se presenta un esquema analítico que vincula el nivel alcanzado por las competencias de los trabajadores tanto con el sistema educativo como con otros factores, entre ellos el papel de la demanda (a través del análisis de los proyectos en que participan)

\footnotetext{
${ }^{11}$ El escaso énfasis de las políticas públicas en resolver los problemas de desvinculación entre estos planos se observa no solo en Argentina sino también en otros países de desarrollo similar. Por ejemplo, en Brasil hay más de 20 programas de fomento del sector informático, pero salvo dos que consideran la formación de recursos humanos y la vinculación con la demanda, el resto está dirigido al desarrollo de empresas, a la creación de negocios y a la integración de fondos de capital de riesgo. En Uruguay, aunque el número de programas es menor, se presenta una situación parecida. Estas descripciones contrastan con lo que sucede en India, donde se abarcan todos estos temas de manera sistémica y se subraya la importancia de los procesos de formación de competencias en la creación de ventajas para el sector (Anlló, Bezchinsky y otros, 2003).

12 Según Jacovkis (2003) la historia de la computación en Argentina estuvo sensiblemente afectada por los acontecimientos políticos sucedidos en el país entre 1966 y 1983. Por más que se intente utilizar criterios neutros, no es posible tener una visión adecuada de la realidad si no se tiene en cuenta el daño terrible que provocaron las dictaduras militares a su incipiente desarrollo.
}

y de las redes en que se integran. Vemos así que las posibilidades de desarrollo del sector se sustentan en las competencias individuales de los trabajadores, pero también en los diversos agregados y sistemas de los que forman parte. Esta manera de enfocar el problema remite a diversos autores y corrientes de pensamiento que consideran que la competitividad es un fenómeno sistémico (Esser, Hillebrand y otros, 1996). ${ }^{13}$

Diversos autores ponen de manifiesto a su vez que, debido a las características inherentes a este tipo de labor, los trabajadores informáticos presentan una gran heterogeneidad que se manifiesta en diferencias de productividad entre programadores de similar formación (Cusumano, 2000). La definición de los perfiles de los trabajadores no dependería únicamente del tipo de tecnología con la cual trabajan o de los productos o servicios que desarrollan, sino que se asociaría también a otros factores, como: i) el avance del sistema de ciencia y tecnología; ii) la articulación de trabajadores en asociaciones gremiales y técnicas y en instituciones académicas; iii) las características y el volumen de

\footnotetext{
13 Sobre el caso específico de las TIC, véase National Research Council (1998).
} 
la demanda local, y iv) el sendero evolutivo de la informática (Castells, 1996). ${ }^{14}$

El gráfico 1 muestra una estilización de la estructura de relaciones posibles entre las diversas dimensiones mencionadas. Desde una perspectiva teórica, en las situaciones más virtuosas existiría una fuerte interrelación de todas ellas: la oferta del sistema educativo y el perfil de la demanda interna y externa -condicionada por el perfil de especialización productiva y el tipo de agentes dominantes- desempeñan un papel clave en la complejidad de los proyectos y, por lo tanto, en el nivel de las competencias técnicas de los trabajadores informáticos.

En este contexto, resulta interesante indagar en qué medida la estructura de la demanda y la complejidad de los proyectos de desarrollo influyen sobre las posibilidades de aprendizaje de los trabajadores, bajo el supuesto de que, ante actividades complejas, ellos enfrentan desafíos que les permiten aprender a través de la experiencia y la interacción con otros agentes (procesos co-

GRÁFICO 1

Argentina: Estructura de las relaciones entre los factores que caracterizan los perfiles de los trabajadores informáticos

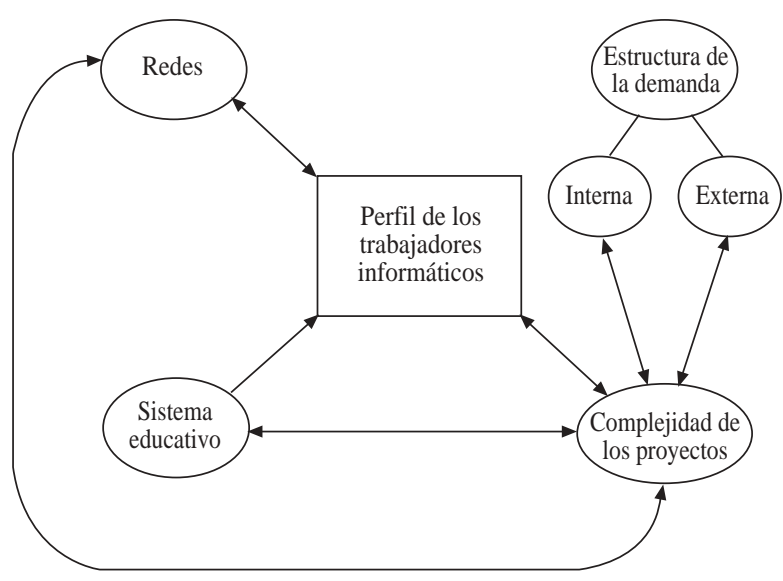

Fuente: Elaboración propia.

14 Castells (1996) argumenta que emerge una nueva división del trabajo definida en función de tres dimensiones: las actividades que generan valor, las que generan relaciones con el entorno y las que afectan el proceso de decisiones. Esas dimensiones dan lugar a tres tipologías superpuestas de trabajadores, que son las siguientes: i) "comandantes", investigadores, diseñadores, integradores, operadores y "operados" (en la dimensión de actividades que generan valor); ii) trabajadores en red, trabajadores "enredados" y trabajadores desconectados (en la dimensión de las relaciones), y iii) los que deciden, los que participan y los que ejecutan (en la dimensión de las decisiones). nocidos como "learning by doing" y "learning by interacting").

Si bien las actividades informáticas tienen lugar dentro de empresas e instituciones, una gran parte de ellas se organiza cada vez más en proyectos que involucran a personas de diversas empresas y a tecnólogos e investigadores vinculados a universidades y laboratorios estatales y privados. Esta escala de proyecto es particularmente apropiada para medir en un plano más agregado, pero aún intermedio, las competencias de los trabajadores y de las empresas e instituciones, y para estudiar actividades que hacen uso intensivo de conocimiento e información (Lam, 2002), bajo el supuesto de que el grado de desarrollo de las redes virtuales, institucionales y personales tiene una influencia decisiva en la circulación del conocimiento codificado y tácito. En los casos virtuosos en los que las redes son muy importantes se generan "comunidades epistémicas" que facilitan los procesos de innovación, poniendo de manifiesto que éstos no constituyen un fenómeno individual (David, Cowan y Forey, 2000). En el caso particular de la informática, el desarrollo de Internet y de las redes virtuales aparece como una herramienta fundamental para la comunicación y la formación de competencias. ${ }^{15}$

En lo que se refiere a la relación entre las competencias técnicas de los trabajadores informáticos y el nivel de educación formal alcanzado, la literatura muestra que éste es un nexo problemático que debe ser estudiado en función de sus connotaciones conceptuales y de política. Se ha señalado que, en una gran proporción de los trabajadores informáticos, la capacidad de utilizar con eficacia y creatividad sus conocimientos y habilidades -manipulación de datos y palabras, representaciones orales y visualestiene más importancia que las credenciales acerca de su nivel educativo y campo de estudios (López-Bassols, 2002; Micheli, 2003).

La relevancia de la relación entre la universidad y el sector informático va más allá de cuestiones de formación, y tiene que ver con aspectos como la investigación conjunta o el establecimiento de redes formales o informales que conecten a empresas, universidades y otras instituciones (Hetzkowitz y Leydesdorf, 1997).

\footnotetext{
${ }^{15}$ Un ejemplo de comunidad epistémica en el sector informático es la comunidad de creadores y usuarios de software libre. Esta se sustenta en la existencia y reproducción de una red de conocimiento, sobre la estructura de Internet, alimentada por colaboraciones sin más barrera de entrada que los requerimientos mínimos de competencia (Dale y Julien, 2003).
} 
La literatura sobre el tema ha señalado la importancia de las "instituciones puente" (Casalet, 2003), capaces de articular ofertas y demandas y de crear mercado.

Como consecuencia, en las actividades estrechamente vinculadas a investigación y desarrollo, diversas empresas están operando con un modelo de red que permite conectar la base interna de conocimiento que posee una empresa, con nuevo conocimiento generado en redes que atraviesan los límites organizacionales (Lam, 2002). Según Lam, las actividades de innovación en los sectores de alta tecnología se organizan crecientemente a través de alguna forma de red, e incentivan en particular las relaciones universidadempresa.

Entre las redes que actúan en la circulación del conocimiento y la formación de competencias, se destacan las de contactos personales, las virtuales y las que pertenecen a instituciones que agrupan a los trabajadores informáticos. Como temas de investigación, han figurado en años recientes en algunos análisis de las actividades vinculadas con la información y el conocimiento. Muchos de esos trabajos se ocuparon de las redes formales e informales en sistemas locales de alto desarrollo, como Silicon Valley (Brown y Duguid, 2000; Castilla, Hwang y otros, 2000; Saxenian, 2000). ${ }^{16}$ Según esos y otros estudios lo relevante ya no es reconocer la importancia de esas redes sino comprender su naturaleza y funcionamiento.

En el caso especial de los países de menor desarrollo relativo, las relaciones teóricas sugeridas en el gráfico 1 son menos evidentes. Así, por ejemplo, en Argentina la demanda interna de las empresas de menor tamaño se dirige a la oferta nacional de software, en el marco de una muy débil inserción externa del sector informático. Por otro lado, la demanda de software y servicios informáticos importados hace que no se busque solución local a innumerables problemas de los sectores más dinámicos de la economía, que sí podrían ser resueltos por la industria informática argentina. ${ }^{17}$ Todo esto explica por qué los proyectos infor-

\footnotetext{
16 El estudio de la dimensión social de la actividad económica (Granovetter, 1985) ha generado numerosos trabajos sobre los diversos tipos de redes personales e institucionales existentes en la actividad económica. Entre ellos se hallan los estudios sobre las redes de inmigrantes en Silicon Valley (Saxenian, 2000) y los estudios sobre la diáspora y su potencial contribución al desarrollo. 17 Por ejemplo, los vinculados a la "trazabilidad" (aseguramiento de la calidad) en la industria agroalimentaria, la circulación del
}

máticos que se llevan adelante localmente siguen siendo de baja calidad. El sistema educativo, por lo demás, tiene una relación poco intensa y más bien unidireccional con los proyectos informáticos de desarrollo, mientras que las redes se vinculan tenuemente con el sistema educativo y en forma más directa con la complejidad de los proyectos. Esto tiene particular importancia en el caso de las redes personales y, en menor medida, en el caso de las redes virtuales e institucionales. Así, las relaciones entre los planos mencionados pierden sistematicidad y condicionan el nivel y la utilización de las competencias de los trabajadores informáticos.

Desde la perspectiva analítica utilizada, la hipótesis central de este trabajo es que coexisten diferentes perfiles de trabajadores informáticos en función de sus competencias tecnológicas. Esos perfiles estarían determinados por los siguientes elementos: las herramientas que dominan (tecnologías y lenguajes), las actividades que realizan (de servicios y de desarrollo), la complejidad de los proyectos en que participan, el grado de utilización de redes y el nivel de educación formal en informática.

La conformación de los grupos de trabajadores informáticos según su nivel de competencias estaría condicionada por la evolución del sector y, en particular, por las trayectorias laborales de cada uno de estos grupos. Así, en el grupo con mayores competencias cabría esperar la presencia de recursos humanos cuyas trayectorias laborales se hayan nutrido de las experiencias más exitosas del sector en las últimas décadas, o bien la presencia de recursos humanos más jóvenes que se hayan formado con los anteriores.

La educación formal de los trabajadores informáticos no bastaría para explicar sus competencias acumuladas, lo cual se reflejaría tanto en la presencia de ocupaciones que exigen una menor calificación (hipótesis de devaluación educativa) como en el caso opuesto (hipótesis de la importancia de la autoformación y del conocimiento tácito). En este marco, la evolución de las competencias de los trabajadores informáticos estaría condicionada por el nivel inicial de esas competencias, por el papel del sistema educativo en su formación y por el grado de complejidad de la demanda.

conocimiento en redes, la codificación del conocimiento tácito, la internalización del conocimiento codificado y otros. 


\section{IV}

\section{Principales resultados de la encuesta y evaluación de la hipótesis}

En esta sección se presentan las principales características del panel de trabajadores informáticos encuestados y se evalúa la hipótesis central del trabajo. Para esto último se consideran conjuntamente las variables asociadas a ella, aplicando el análisis factorial de correspondencias múltiples a los principales indicadores surgidos de la encuesta (véase el apéndice A). Este instrumento de técnica estadística se aplica aquí fundamentalmente para examinar los factores que contribuyen a explicar las competencias tecnológicas desde una perspectiva sistémica.

El cuestionario de la encuesta fue elaborado a partir de un trabajo interdisciplinario realizado por profesionales (principalmente economistas) de la Universidad Nacional de General Sarmiento y por profesionales informáticos de la Sociedad Argentina de Informática e Investigación Operativa (sADIO). Fue necesario un exigente proceso de adaptación que compatibilizara las hipótesis del trabajo con las formas de captar información en el sector sin perder su complejidad. ${ }^{18}$ El cuestionario, que se administró electrónicamente, contenía las siguientes secciones, referidas a: i) la situación laboral de los entrevistados; ii) la organización en la que trabajan; iii) las actividades específicas que llevan a cabo; iv) las características de los proyectos de desarrollo que realizan; v) el uso y creación de software libre; vi) las vinculaciones institucionales y la participación en redes, y vii) la educación formal e informal.

El panel encuestado incluyó trabajadores informáticos de empresas privadas, del sector público, de centros de capacitación, de consultoras especializadas y de universidades. Se les invitó a contestar el cuestionario electrónico a través de diversos medios. La encuesta se realizó entre noviembre del 2003 y marzo del 2004 y participaron 197 trabajadores. Luego de analizar y filtrar las respuestas quedaron 167 registros, sobre los cuales se realizó el análisis estadístico.

\footnotetext{
${ }^{18}$ El cuestionario puede consultarse en www.littec.ungs.edu.ar.
}

\section{Las principales características del panel encuestado y las variables de corte}

El panel consultado presenta algunos rasgos principales en cuanto a género, edad y localización geográfica. En él predominan los hombres jóvenes, con una proporción significativa de menores de 40 años (80\% del panel). La mayoría habita en el Polo Metropolitano (58\%), en el resto de la Provincia de Buenos Aires (11\%), en Córdoba y Santa Fe (9\% en cada caso), y el $8 \%$ en otras provincias argentinas. Finalmente, un 5\% de los encuestados corresponde a argentinos residentes en el exterior.

La mayor parte de los entrevistados tiene un elevado nivel de calificación. Mientras la mitad posee formación universitaria completa, un $17 \%$ tiene posgrados que se vinculan con la informática y algo más de un cuarto posee estudios universitarios incompletos. Entre los que tienen títulos universitarios, el $60 \%$ corresponde a ingenieros y licenciados en computación, el $29 \%$ a analistas y licenciados en sistemas y solo el $11 \%$ tiene estudios no vinculados con la informática. Además, estas calificaciones se completan con un $30 \%$ de entrevistados que poseen algún tipo de certificación de las empresas que proveen las tecnologías más utilizadas y casi tres cuartos del panel que tienen conocimiento de inglés bueno o superior.

La tasa de entrevistados económicamente activos es muy alta: sólo el 1,2\% está desempleado. En general, son personas con menos de diez años de trabajo en informática (50\% del panel), lo que está fuertemente asociado a su edad. Solo el $10 \%$ trabaja en esta actividad desde hace más de 25 años. Un rasgo predominante es la gran heterogeneidad de la tasa de rotación de los encuestados en sus puestos. Mientras en los últimos cinco años prácticamente la mitad del panel no cambió de trabajo, un quinto ha cambiado de empleo al menos tres veces, y el $40 \%$ del panel tiene menos de dos años de antigüedad en su trabajo actual.

En cuanto a la categoría ocupacional de los entrevistados, la mayor parte se encuentra empleada en relación de dependencia (57\%), algo menos de un quinto es contratado por obras o servicios y el resto es 
propietario. Entre aquellos que se encuentran en relación de dependencia, la mayor parte trabaja en empresas privadas (60\%), algo menos de un quinto en el sector público (nacional, provincial o municipal) y el resto en instituciones académicas o tecnológicas o en organizaciones no gubernamentales. Por el contrario, entre aquellos a contrata el peso del Estado y de las empresas privadas es relativamente similar $(45 \%$ y $42 \%$, respectivamente).

El 40\% de los encuestados trabaja en empresas cuya actividad principal es la informática y el $60 \%$ en empresas u otro tipo de organizaciones que se dedican a otra actividad. Entre los primeros, la mayor parte $(84 \%)$ se dedica a actividades de desarrollo y a servicios informáticos, el $6 \%$ solo a servicios y el $10 \%$ restante únicamente a actividades de desarrollo.

Finalmente, respecto a las variables utilizadas para la evaluación de la hipótesis, cabe señalar que algo menos de un quinto de los entrevistados no domina lenguajes ni tecnologías de la informática. Sin embargo, la proporción de trabajadores informáticos que no usa esos lenguajes es aún mayor, lo que pone de relieve problemas de subutilización. En lo que respecta al uso de redes, cabe destacar la importancia de las redes de contactos personales; más de la mitad de los encuestados indica que consiguió su puesto actual a través de ellas y el $78 \%$ del panel las utiliza para enterarse de los adelantos tecnológicos. Los instrumentos de comunicación por Internet que más se utilizan son los foros especializados, seguidos por los boletines (newsletters) y las listas de correo. Los trabajadores locales hacen poco uso de herramientas como el IRC (Internet related chat). Finalmente, casi el 30\% del panel no está involucrado en proyectos de desarrollo. Los proyectos suelen ser de poca duración (el 50\% de ellos dura menos de un año) e involucran un bajo número de personas (menos de 10 personas en el $76 \%$ de ellos), lo que pone de manifiesto su escasa complejidad.

\section{Análisis de grupos (clusters analysis)}

La aplicación del análisis de grupos permitió constituir grupos homogéneos sobre la base de considerar simultáneamente los diversos factores que influyen en las competencias técnicas de los trabajadores informáticos. Utilizando el análisis factorial de correspondencias múltiples (AFCM) fue posible sintetizar las asociaciones existentes entre las diferentes modalidades de las variables que componen la matriz de datos (variables activas), y obtener un conjunto limitado de grupos compuestos por individuos que presentan una alta homogeneidad intragrupo y una elevada heterogeneidad extragrupo. ${ }^{19} \mathrm{~A}$ su vez, para enriquecer la descripción de ejes y grupos, se tuvieron en cuenta variables ilustrativas no consideradas al momento de determinar los ejes factoriales.

En el análisis de grupos se incluyeron siete variables activas: i) la complejidad de los lenguajes que domina el encuestado; ii) la complejidad de las tecnologías que domina; iii) la complejidad de las actividades de desarrollo que realiza; iv) la complejidad de los servicios que presta; v) el nivel de educación formal alcanzado; vi) la complejidad de los proyectos en los que ha participado, y vii) la participación en redes (véase el apéndice A). Se consideraron además diversas variables ilustrativas, que se recogen en el apéndice $\mathrm{B}$, como tipo de carrera y nivel del encuestado (cuadro B.1), ámbito en el que considera que obtuvo la mayor parte de sus competencias (cuadro B.2), tipo de organización en la que desarrolla sus actividades (cuadro B.3), rotación laboral (cuadro B.4), edad y años de experiencia laboral (cuadros B.5 y B.6) y género (cuadro B.7). A pesar de que la evolución del sector es fundamental para determinar los perfiles de los trabajadores informáticos, la dificultad de incorporar tal información a un análisis de grupos condujo a que este factor se considerara únicamente a título contextual y descriptivo.

A partir del AFCM, se realizó el análisis de grupos que permitió identificar cinco grupos homogéneos. ${ }^{20}$ Los grupos homogéneos pueden ordenarse en función del nivel de las competencias tecnológicas alcanzadas: muy bajo, bajo, medio, alto y muy alto.

La posibilidad de reunir a los individuos en diferentes grupos homogéneos, caracterizados por combinaciones de modalidades coherentes que pertenecen a distintas variables, refleja la fuerte interdependencia de las dimensiones mencionadas en la hipótesis. Así, puede interpretarse que esas dimensiones contribuyen a explicar los diferentes niveles de competencias tecnológicas en los trabajadores informáticos. A

\footnotetext{
${ }^{19}$ Este método opera mediante la reducción del número de variables del fenómeno estudiado, conformando ejes factoriales cuya determinación permite concentrar el análisis en aquellas variables y modalidades que más contribuyen a explicar la problemática abordada, brindando así una visión manejable de ella.

${ }^{20}$ Para interpretar los resultados, debe tenerse en cuenta que las modalidades de las variables que se encuentran asociadas a un determinado grupo indican que los individuos que tienen esa característica están significativamente más representados en el grupo que en el total de la muestra, lo que no implica necesariamente que todos los individuos de dicho grupo presenten esa característica.
} 
continuación se describen los grupos enumerados en el párrafo anterior.

\section{a) Grupo con competencias tecnológicas de nivel muy bajo \\ En este grupo, que representa el $18 \%$ del panel,} predominan las tareas de servicios (el 53\% de sus integrantes no realiza actividades de desarrollo), el escaso dominio de lenguajes y tecnologías informáticos (el $80 \%$ del grupo no domina tecnologías y el $67 \%$ no domina lenguajes) y la no utilización de redes virtuales (el $30 \%$ no utiliza redes, y $60 \%$ de los que no utilizan redes están en este grupo). Entre las variables ilustrativas (es decir, las que no se emplearon en el análisis de grupos), se puede mencionar un conjunto de características ${ }^{21}$ que resultan útiles con fines descriptivos: se trata de trabajadores de más de 40 años, que en promedio están hace cuatro años y medio en la empresa, pero cuya antigüedad varía entre menos de dos años y más de cuatro (cuadros B.4, B.5 y B.6).

Las personas de mayor edad en el grupo exhiben dos trayectorias laborales posibles. Una de ellas, la más virtuosa, es la de quienes participaron activamente en los mayores avances del sector en las últimas décadas y que estuvieron vinculadas principalmente a actividades científicas y tecnológicas; actualmente se desempeñan en instituciones fundamentalmente académicas. La otra trayectoria laboral, recorrida por la mayor parte de los trabajadores del grupo, corresponde a personas que se acercaron a la informática a través de su actividad laboral y tuvieron una formación estrictamente funcional al ámbito de trabajo en el que se desempeñaban. ${ }^{22}$

Es característica central de este grupo, por lo tanto, la de tener formación universitaria no vinculada a la informática (cuadro B.1), escaso o nulo conocimiento del inglés y haber adquirido su formación en trabajos anteriores. En este contexto, ser autodidacta no es un rasgo típico del grupo (cuadro B.2). Sus integrantes tienen bajas capacidades en materia de análisis y programación, y debido al escaso peso entre ellos de las actividades de desarrollo efectúan tareas que no están organizadas en proyectos. Asimismo, se desempeñan en los sectores de comercio y servicios privados.

\footnotetext{
${ }^{21}$ Se consideran rasgos característicos si en la tabla de contingencia la diferencia entre la proporción del atributo en el grupo y en el total del panel (prueba Z) es significativamente distinta de 0 (al $10 \%$ de significación como máximo).

${ }^{22}$ En ese sentido, su formación se fue limitando exclusivamente al dominio de herramientas.
}

Otros rasgos que merecen ser mencionados, pese a no ser característicos, son la pertenencia a empresas cuya actividad principal no es la informática (57\%) y una mayor proporción relativa de mujeres (cuadro B.7) que en los demás grupos (27\%).

\section{b) Grupo con competencias tecnológicas bajas}

Constituye el más numeroso del panel (39\%). Está integrado por trabajadores que no dominan lenguajes y tecnologías informáticas o dominan herramientas de baja complejidad ${ }^{23}$ y realizan tareas de desarrollo del mismo nivel. Sus actividades no están organizadas en proyectos. En él predominan personas con formación universitaria incompleta.

Entre los rasgos característicos del grupo destacan la prevalencia de gente joven (entre 20 y 30 años), que cuenta con menos de 10 años de experiencia laboral (cuadros B.5 y B.6) y que, en promedio, ha permanecido en el trabajo cuatro años y medio (cuadro B.4). Se trata de personas que se incorporaron recientemente al mercado laboral y que, en un porcentaje significativo, se desempeñan en el sector público (cuadro B.3). Casi las tres cuartas partes de los miembros del grupo trabajan en organizaciones cuya actividad principal no es la informática. En cuanto a la trayectoria de las personas de este grupo, puede pensarse que están en un momento de su formación en que, o bien pueden evolucionar hacia los grupos con competencias de nivel medio y alto, o estancarse, lo que implicaría pasar a formar parte del grupo anterior.

\section{c) Grupo con competencias tecnológicas de nivel medio}

Este grupo, que abarca el 10\% de los trabajadores informáticos del panel, es el más pequeño. Sus integrantes tienden a realizar tareas y a dominar tecnologías de complejidad media en actividades de desarrollo. Es el primer grupo que se destaca por el dominio de lenguajes y tecnologías informáticos y por participar en proyectos de desarrollo. Sus integrantes tienen educación universitaria completa en el área de la informática (cuadro B.1).

Entre los rasgos característicos del grupo se hallan la poca edad y la alta rotación. Las personas que lo componen son menores de 30 años y la mitad lleva menos de tres años en la organización para la que

\footnotetext{
23 Visual Basic, Clipper y Cobol entre los lenguajes; plataforma web y proceso unificado entre las tecnologías.
} 
trabaja. A su vez, casi el $40 \%$ cambió tres veces de trabajo en los últimos cinco años (cuadro B.4). Si bien los miembros de este grupo participan en proyectos, estos no se caracterizan por ser muy complejos. Se trata de proyectos relativamente pequeños (ocupan de 9 a 16 personas), realizados básicamente para terceros, y responden a encargos específicos (esto es, son productos a la medida y no para ser comercializados a varios clientes o en forma masiva). Desde la perspectiva de los entrevistados, la criticidad ${ }^{24}$ de los proyectos es elevada y la complejidad de las competencias técnicas requeridas es media. A su vez, la realización de ellos requirió vinculaciones con grandes empresas de software y servicios informáticos. Se puede establecer una relación entre la naturaleza de los proyectos y la naturaleza de la demanda diversificada de varios sectores.

Una característica de este grupo, ausente en los anteriores, es la pertenencia a empresas que se dedican a la informática como actividad principal: algo más de la mitad de sus integrantes (56\%) trabaja en tales empresas.

\section{d) Grupo con competencias tecnológicas de nivel alto}

Este grupo corresponde al 15\% del panel. Las actividades de desarrollo y de servicios que realizan sus integrantes son de menor complejidad que las herramientas que dominan. Desde el punto de vista de las variables ilustrativas, entre ellos predominan los trabajadores menores de 30 años (cuadro B.6). La rotación es muy elevada, comparable a la del grupo anterior, pero la permanencia en el trabajo actual es significativamente menor, lo que se asocia directamente con la edad: la mitad ha estado menos de nueve años en el mercado laboral (cuadros B.4 y B.5). Si bien la educación formal no constituye un factor característico, casi la mitad de los integrantes tiene educación universitaria vinculada a la informática, aunque no todos han terminado la carrera (cuadros B.1 y B.2). Un elemento que distingue a estas personas es su elevado conocimiento del inglés. Los proyectos de desarrollo en que participan aparecen como relativamente inferiores a los del grupo anterior. Esto se manifiesta en lo siguiente: los proyectos duran menos de un año e involucran entre cinco y ocho persona; la criticidad y

\footnotetext{
${ }^{24}$ Según lo indicado en el formulario de la encuesta, la criticidad del software se entiende en relación con el objetivo al que está destinado: por ejemplo, la severidad del daño que podría producirse ante una eventual falla.
}

complejidad de las competencias técnicas requeridas son evaluadas por los entrevistados como bajas y, finalmente, no se requirieron muchas vinculaciones con grandes empresas de software y servicios informáticos. La mayor parte del grupo trabaja en empresas privadas cuya actividad principal es la informática, y destaca como rasgo característico la presencia de propietarios. Cabría identificar este grupo como subutilizado (hipótesis de devaluación educativa), ya que los trabajadores que lo componen poseen competencias técnicas significativamente superiores al grado de complejidad de las tareas que se les encomiendan. Es posible que este desequilibrio los desaliente en su trabajo, lo que explicaría su alta tasa de rotación.

\section{e) Grupo con competencias tecnológicas de nivel muy alto \\ Por último, el 18\% restante de los trabajadores} informáticos pertenece a este grupo con competencias más complejas. ${ }^{25}$ Tanto las actividades que realizan sus integrantes como los lenguajes y tecnologías que dominan son los más avanzados. Por otra parte, hacen uso intensivo de redes virtuales, tienen educación formal de posgrado y llevan a cabo proyectos de alta complejidad (cuadro B.1). Los rasgos característicos del grupo son: el predominio de edades intermedias (entre 31 y 40 años), la muy escasa rotación laboral y una permanencia en el actual trabajo mucho mayor que en los demás grupos: siete años en promedio, con un $50 \%$ que lleva más de cinco años en el puesto (cuadros B.4, B.5 y B.6). Este rasgo, junto con el nivel de educación formal señalado y un excelente dominio del inglés, son factores que determinan el alto grado de competencias tecnológicas que exhiben los incluidos en este grupo. A la vez, la calidad de los proyectos en los que trabajan y el uso intensivo de redes contribuyen a que sus competencias se incrementen a lo largo del tiempo. Los proyectos en que participan duran más de 18 meses, ${ }^{26}$ involucran a más de nueve personas, presentan gran dificultad técnica y requieren recursos humanos con altas capacidades. La realización de este tipo de proyectos implica establecer importantes vinculaciones con grandes empresas de software y servicios informáticos, lo que marca otra diferencia significativa de este grupo frente a los demás. Un rasgo característico es que sus integrantes ocupan posiciones permanentes

\footnotetext{
${ }^{25}$ Lenguajes como C++, PHP, Perl, Lisp y Haskel, entre otros.

${ }^{26}$ Esta duración corresponde al 54\% de los proyectos en el grupo, y solo al $32 \%$ de los proyectos en el total del panel.
} 
en instituciones académicas o de investigación. No es extraño entonces que la demanda de sus servicios provenga del sector informático y del de investigación y desarrollo (cuadro B.3). Como se dijo antes, las trayectorias laborales de los trabajadores de este grupo se han alimentado de las experiencias más virtuosas de la evolución del sector informático, a pesar de sus marchas y contramarchas.

Otras dos características distintivas de este grupo son una elevada proporción de residentes en el exterior $(20 \%)$ y una baja proporción de mujeres $(13 \%)$ (cuadro B.7). Se abren, en esa línea, diversos interrogantes. Cabe preguntarse, de un lado, qué rol podrían desempeñar los trabajadores informáticos argentinos radicados fuera del país en el desarrollo del sector en Argentina. Y de otro, por qué la proporción de mujeres es reducida en el sector informático en general y particularmente en los grupos con elevadas competencias tecnológicas.

\section{La dinámica de los distintos grupos}

Desde una perspectiva evolucionista, resulta esencial incorporar algunas variables dinámicas que expliquen la evolución de las competencias de los individuos (Freeman, Soete y Efendioglu, 1995; Lall, 2001). Con este fin se midió en cada grupo, para 1991 y 2004, la proporción de recursos humanos que, o bien no realizaban actividades de desarrollo o servicios, o bien sus actividades de esa índole eran de muy baja calidad.

En términos generales, se detectaron diferencias entre los grupos respecto a la evolución de tales actividades entre 1991 y 2004. En los grupos con compe- tencias tecnológicas de nivel bajo o muy bajo tendió a aumentar la proporción de personas que se desempeñaban en actividades de muy baja complejidad, tanto de servicios como de desarrollo: mientras que en 1999 el $61 \%$ de sus integrantes se dedicaba a actividades de desarrollo de baja complejidad, en el 2004 la cifra ascendía a $79 \%$. Por el contrario, en el grupo con competencias tecnológicas de nivel muy alto la evolución fue muy positiva: mientras que en 1999 casi la mitad no llevaba a cabo actividades de desarrollo complejas, hacia el 2004 esa proporción se había reducido al 16\%.

Esta mirada a la dinámica de las trayectorias laborales permite extraer algunas conclusiones de interés para la reflexión sobre las competencias de los recursos humanos informáticos en Argentina. Se comprueba que en el proceso de acumulación de capacidades hay significativos bloqueos que la dinámica del mercado de trabajo y las actividades dentro de las empresas no parecen resolver, ni para los trabajadores con menos educación formal y los que realizan tareas más simples, ni para un grupo con elevadas competencias que no puede mejorar la calidad de las tareas que ejecuta. Por lo tanto, la subutilización de capacidades no parece ser un fenómeno pasajero sino que, para muchos, es una constante. Cabe, por lo tanto, identificar dos problemas distintos. Uno es el de las personas con bajas competencias tecnológicas que no pueden encontrar una senda evolutiva virtuosa, debido a las limitaciones del sistema institucional, la heterogeneidad del sistema educativo y la poca presión de la demanda. El otro es la persistencia de la subutilización de los recursos, motivada básicamente por la debilidad de la demanda de productos complejos.

\section{V}

\section{Conclusiones y reflexiones finales}

En este trabajo se evaluó la hipótesis de la existencia de distintos perfiles de trabajadores informáticos caracterizados por sus competencias tecnológicas y el modo como estos perfiles se determinan en función de variables como la participación en redes, el sistema educativo, la complejidad de los proyectos en que se participa y la estructura de la demanda. La hipótesis fue validada por medio de un análisis de grupos homogéneos de trabajadores informáticos constituidos a partir de las principales variables que determinan sus competencias tecnológicas. La posibilidad de reunir a los individuos en los diferentes grupos homogéneos se debió a la gran interdependencia de las dimensiones consideradas en la hipótesis y al carácter sistémico de las mismas. Por lo tanto, las dimensiones consideradas en la hipótesis contribuyen a explicar los diferentes niveles de competencias tecnológicas de los trabajadores informáticos.

La estructura de la demanda aparece fragmentaria y no especializada. Se registran pocas ventas a los 
sectores más dinámicos de la economía, que son los que podrían plantear demandas más complejas. Los principales demandantes son el comercio y los servicios en general, pero tanto en estos sectores como en la industria manufacturera la demanda dirigida al mercado local es de menor complejidad que la orientada a los mercados externos. Esto influye en el tipo de proyectos que se llevan a cabo y en la formación de las competencias de los trabajadores informáticos, y a la vez genera subutilización de estos trabajadores. Así, a los sectores informáticos internos se les pide sobre todo adaptaciones para incorporar las especificidades locales, siendo que con demandas más complejas por parte de los sectores más dinámicos de la economía nacional se incentivaría a las empresas informáticas locales a resolver problemas más complejos, acordes con la presión competitiva que enfrentan. El sector productivo y el sistema institucional todavía tienen dificultades para expresar sus requerimientos de manera que la oferta local pueda decodificarlos y satisfacerlos adecuadamente.

Por otro lado, se observa una escasa participación de los trabajadores informáticos en redes virtuales e institucionales, siendo que este tipo de vinculaciones podría generar competencias que complementaran las calificaciones obtenidas en el sistema de educación formal. Las redes personales aparecen relativamente más utilizadas, fundamentalmente en la búsqueda de actualización tecnológica o la obtención de empleo; este predominio condiciona la circulación de conocimiento al despliegue de esfuerzos individuales. Por lo tanto, el potencial para la autoformación y para la creación de capacidades individuales y grupales que ofrecen las redes no es aprovechado de manera cabal y sistemática por las instituciones, que son las que podrían actuar sobre un conjunto más amplio de personas, empresas y otras organizaciones.

Si bien existe una asociación positiva entre la pertenencia al grupo con mayores competencias y el nivel de educación formal alcanzado, la formación en el trabajo y la autoformación aparecen como factores que contribuyen a construir las competencias de los trabajadores. El sistema de educación formal sería el encargado de generar un umbral mínimo y, a partir de allí, los trabajadores estarían en condiciones de internalizar los conocimientos adquiridos en los procesos de aprendizaje en el trabajo y a través de las relaciones que establecen y se generan en su propio proceso de formación.

La encuesta revela que el mercado laboral informático en Argentina adolece de problemas que obsta- culizan procesos de perfeccionamiento y el paso hacia los grupos con mayores competencias tecnológicas. Habría impedimentos para que los que saben lleven a cabo las actividades para las cuales están preparados, y para que quienes dominan herramientas simples pasen a utilizar herramientas complejas.

Por un lado, hay capacidades latentes o potenciales que no son aprovechadas a cabalidad, debido a las debilidades de la oferta y de la demanda ya señaladas. Por otro lado, en los grupos que realizan actividades simples se observa una dinámica limitada e incapacidad para la autoformación. Muchos de los trabajadores con bajas competencias tecnológicas tienen pocas oportunidades de pasar a los grupos más virtuosos, por carecer del umbral de competencias mínimo que les permita autoformarse mediante la internalización del conocimiento codificado existente. Por lo tanto, parece necesario mejorar el proceso de generación de las capacidades informáticas, de modo de incentivar un círculo virtuoso de interacción de la oferta y la demanda.

Hemos presentado aquí una tipología de las personas que trabajan en el sector informático en Argentina, la que es el resultado de un complejo proceso histórico que involucra construcción (y destrucción) de instituciones y capacidades técnicas en el marco de distintos intentos estatales y privados de impulsar los incipientes avances nacionales en informática. Esta tipología refleja al menos en parte esos tiempos pasados y las contradictorias intenciones de la sociedad argentina de construir un sector informático dentro de sus fronteras.

La tipología presentada y examinada en este trabajo, así como la descripción y el análisis de la historia de la informática en Argentina, plantean algunos interrogantes sobre las potencialidades del sector informático argentino en general y de sus recursos humanos en particular. Resulta difícil sostener que estamos ante una inminente y espectacular expansión de la oferta exportable de software, aunque de hecho haya habido recientemente un importante aumento de la oferta exportable de servicios informáticos. En este sentido, cabe sugerir algunos ejes de acción a partir del análisis efectuado.

En lo que se refiere a los proyectos, las acciones deberían centrarse en i) elevar el umbral general de complejidad de los proyectos de software y servicios informáticos que el país puede acometer; ii) acrecentar las relaciones entre el sector informático y las actividades más dinámicas, como la industria, el agro y la minería; iii) fomentar la demanda de software y servicios informáticos derivada de las empresas trasnacionales radicadas en Argentina, y iv) aprovechar 
la experiencia, contactos y conocimiento de los profesionales informáticos argentinos en el exterior.

Por el lado de las instituciones y de las relaciones con el sector educativo, parece necesario mejorar la institucionalidad del sector informático y promover acciones que permitan incrementar sus relaciones con el sistema educativo.

En cuanto a acciones más específicas para desarrollar capacidades técnicas en los recursos humanos dedicados a la informática, parece relevante i) superar las barreras que hoy existen en el mercado laboral para

APÉNDICE A

Construcción de indicadores

\section{Herramientas que domina el encuestado}

Los lenguajes y las tecnologías informáticos fueron jerarquizados de acuerdo con cuatro criterios: actualidad, importancia en el mercado local e internacional, relevancia académica y perspectivas futuras. Cada lenguaje y cada tecnología recibió la puntuación exponencial $(2,4$ y 8) que mejor respondía a la variabilidad, atendidos los criterios mencionados. Para cada individuo se calculó el promedio de los puntajes obtenidos, considerando el no dominio como cero. El indicador de capacidades surgió del promedio simple del indicador de complejidad de los lenguajes y las tecnologías.

\section{Actividades que realiza el encuestado}

Siguiendo un procedimiento similar, las tareas que se realizan fueron agrupadas en actividades de desarrollo y de servicios y se les otorgó un puntaje en escala exponencial. Este indicador se definió también como un promedio simple de los indicadores de actividades de desarrollo y de servicios. La diferencia fundamental entre el indicador de capacidades de acuerdo a las herramientas dominadas y a las actividades realizadas radica en que para el segundo caso no se otorgó el puntaje de cero en caso de no realizarse determinada actividad. De tal forma, mientras que se consideró positivo que una persona dominara más de una herramienta, el indicador de actividades no premió el hecho de que una persona realizara más de una actividad a la vez.

\section{Indicador del grado de utilización de redes}

La información de la encuesta indicaba el grado de utilización de cada instrumento de comunicación virtual con un puntaje que variaba de uno a cinco: se asignaba uno a la no utilización y cinco a la mayor frecuencia de utilización. El aprovechar todas las capacidades existentes y para promover verdaderas carreras o sendas evolutivas que eleven el nivel general de los recursos humanos informáticos en el país y ii) formular y aplicar políticas específicas que partan de la heterogeneidad que se observa en los perfiles de los trabajadores del sector.

Finalmente, a la visión dinámica de los trabajadores informáticos que se ha ofrecido aquí, sería interesante sumar una visión sistémica que tenga en cuenta los múltiples factores que definen la calidad de estos recursos humanos.

indicador continuo, que varía de uno a cinco, es el promedio de los puntajes para cada uno de los instrumentos.

\section{Indicador de la complejidad de los proyectos}

Este indicador se calculó como un promedio entre i) la duración en meses; ii) el número de personas y iii) el cruce entre el objetivo del proyecto y el mercado de destino. En el cuadro A.1 se detallan estas variables.

CUADRO A.1

Argentina: Variables de los proyectos

\begin{tabular}{|c|c|c|c|}
\hline Puntaje & $\begin{array}{c}\text { Duración } \\
\text { (meses) }\end{array}$ & $\begin{array}{c}\text { Tamaño } \\
\left(\mathrm{N}^{\mathrm{o}} \text { de personas }\right)\end{array}$ & $\begin{array}{l}\text { Objetivo y mercado } \\
\text { de destino }\end{array}$ \\
\hline 1 & $0-6$ & $0-2$ & Interno a la empresa \\
\hline 2 & $7-12$ & $3-4$ & $\begin{array}{l}\text { Por encargo específico de } \\
\text { cliente en Argentina }\end{array}$ \\
\hline 3 & $13-18$ & $5-8$ & $\begin{array}{l}\text { Para ser comercializado en } \\
\text { Argentina }\end{array}$ \\
\hline 4 & $19-24$ & $9-16$ & $\begin{array}{l}\text { Por encargo específico de } \\
\text { cliente en el extranjero }\end{array}$ \\
\hline 5 & 25 y más & 17 y más & $\begin{array}{l}\text { Para ser comercializado en el } \\
\text { extranjero }\end{array}$ \\
\hline
\end{tabular}

El indicador final surge como promedio de los puntajes obtenidos en estas tres variables.

\section{Indicador de educación formal}

El nivel de educación formal de los encuestados fue dividido en cuatro niveles:

i) Educación formal no vinculada a la informática

ii) Educación universitaria incompleta vinculada a la informática

iii) Educación universitaria completa vinculada a la informática

iv) Educación de posgrado vinculada a la informática. 
APÉNDICE B

Información estadística

CUADRO B1

Argentina: Relación entre la educación formal y el nivel de competencias tecnológicas de grupos de trabajadores informáticos ${ }^{a}$

(Porcentajes)

\begin{tabular}{|c|c|c|c|c|c|c|c|}
\hline \multirow[b]{2}{*}{$\begin{array}{l}\text { Nivel de } \\
\text { competencias } \\
\text { de cada grupo }\end{array}$} & \multicolumn{7}{|c|}{ Educación formal clasificada por tipo de carrera y nivel } \\
\hline & $\begin{array}{l}\text { Terciaria } \\
\text { completa }\end{array}$ & $\begin{array}{c}\text { Universitaria } \\
\text { incompleta }\end{array}$ & $\begin{array}{l}\text { Universitaria } \\
\text { no vinculada } \\
\text { a informática }\end{array}$ & $\begin{array}{l}\text { Analistas y } \\
\text { licenciados } \\
\text { en sistemas }\end{array}$ & $\begin{array}{l}\text { Ingenieros y } \\
\text { licenciados en } \\
\text { computación }\end{array}$ & $\begin{array}{c}\text { Universitaria } \\
\text { con posgrado } \\
\text { vinculado a } \\
\text { informática }\end{array}$ & $\begin{array}{c}\text { No } \\
\text { especificada }\end{array}$ \\
\hline 1. Muy bajo & 8,7 & $8,7^{\mathrm{b}}$ & $16,7^{b}$ & 16,7 & 33,3 & 10,0 & $10,0^{\mathrm{b}}$ \\
\hline 2. Bajo & 7,6 & $45,5^{b}$ & 3,0 & 15,2 & 24,2 & $3,0^{\mathrm{b}}$ & 1,5 \\
\hline 4. Alto & 11,5 & 23,1 & 3,8 & 11,5 & 30,8 & 19,2 & 0,0 \\
\hline 5. Muy alto & 6,5 & $9,7^{\mathrm{c}}$ & 0,0 & 6,5 & 25,8 & $48,4^{\mathrm{b}}$ & 3,2 \\
\hline Total & 7,1 & 25,4 & 5,3 & 13,6 & 29,0 & 16,6 & 3,0 \\
\hline
\end{tabular}

Fuente: Elaboración propia con datos de la encuesta SADIO-UNGS sobre las competencias tecnológicas de los trabajadores informáticos.

a La probabilidad de aceptar la hipótesis nula de ausencia de asociación es $0 \%$.

b Prueba $\mathrm{Z}$ significativa al $1 \%$.

c Prueba Z significativa al $10 \%$.

CUADRO B.2

Argentina: Relación entre el ámbito de obtención de competencias y el nivel de competencias tecnológicas de grupos de trabajadores informáticos ${ }^{a}$

(Porcentajes)

\begin{tabular}{|c|c|c|c|c|c|}
\hline \multirow[b]{2}{*}{$\begin{array}{l}\text { Nivel de competencias } \\
\text { de cada grupo }\end{array}$} & \multicolumn{5}{|c|}{ Ámbito en el que el trabajador considera que obtuvo sus principales competencias } \\
\hline & Autodidacta & $\begin{array}{l}\text { Educación } \\
\text { formal }\end{array}$ & $\begin{array}{l}\text { Cursos de } \\
\text { capacitación }\end{array}$ & $\begin{array}{c}\text { Trabajos } \\
\text { anteriores }\end{array}$ & $\begin{array}{r}\text { Trabajo } \\
\text { actual }\end{array}$ \\
\hline 1. Muy bajo & $2,0^{\mathrm{b}}$ & 22,0 & 18,7 & $42,0^{\mathrm{c}}$ & 15,3 \\
\hline 2. Bajo & 16,7 & 27,3 & 10,6 & 19,7 & 25,8 \\
\hline 3. Medio & 20,0 & 20,0 & 13,7 & 26,2 & 20,0 \\
\hline 4. Alto & $23,1^{\mathrm{c}}$ & 34,6 & 15,4 & 19,2 & 7,7 \\
\hline 5. Muy alto & 16,1 & 35,5 & $3,2^{\mathrm{c}}$ & 29,0 & 16,1 \\
\hline Total & 15,3 & 28,3 & 11,7 & 25,9 & 18,8 \\
\hline
\end{tabular}

Fuente: Elaboración propia con datos de la encuesta SADIO-UNGS sobre las competencias tecnológicas de los trabajadores informáticos.

a La probabilidad de aceptar la hipótesis nula de ausencia de asociación es $0,06 \%$.

b Prueba Z significativa al $1 \%$.

c Prueba Z significativa al $10 \%$. 
CUADRO B. 3

Argentina: Relación entre el tipo de organización para la que se trabaja y el nivel de competencias tecnológicas de grupos de trabajadores informáticos ${ }^{a}$ (Porcentajes)

\begin{tabular}{|c|c|c|c|c|}
\hline \multirow[b]{2}{*}{$\begin{array}{l}\text { Nivel de competencias } \\
\text { de cada grupo }\end{array}$} & \multicolumn{4}{|c|}{ Tipo de organización para la que se trabaja } \\
\hline & Empresa privada & $\begin{array}{c}\text { Organización } \\
\text { no gubernamental }\end{array}$ & $\begin{array}{c}\text { Institución académica } \\
\text { o de investigación }\end{array}$ & Sector público \\
\hline 1. Muy bajo & 76,7 & 3,3 & $0,0^{\mathrm{b}}$ & 20,0 \\
\hline 2. Bajo & 49,2 & 4,8 & 7,9 & $38,1^{\mathrm{c}}$ \\
\hline 3. Medio & 75,0 & 6,3 & 6,3 & 12,5 \\
\hline 4. Alto & 88,0 & 0,0 & $0,0^{\mathrm{b}}$ & 12,0 \\
\hline 5. Muy alto & 56,7 & 0,0 & $33,3^{\mathrm{c}}$ & 10,0 \\
\hline Total & 64,0 & 3,0 & 9,8 & 23,2 \\
\hline
\end{tabular}

Fuente: Elaboración propia con datos de la encuesta SADIO-UNGS sobre las competencias tecnológicas de los trabajadores informáticos.

a La probabilidad de aceptar la hipótesis nula de ausencia de asociación es $0 \%$.

b Prueba Z significativa al $10 \%$.

c Prueba $\mathrm{Z}$ significativa al $1 \%$.

CUADRO B. 4

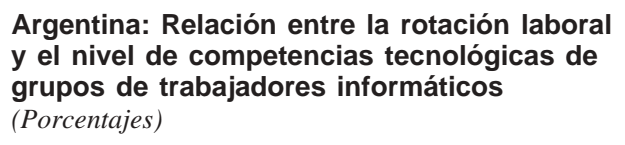

\begin{tabular}{lcccc}
\hline & \multicolumn{4}{c}{ Rotación laboral } \\
\cline { 2 - 5 } $\begin{array}{l}\text { Nivel de } \\
\text { competencias } \\
\text { de cada grupo }\end{array}$ & $\begin{array}{c}\text { No } \\
\text { rota }\end{array}$ & $\begin{array}{c}\text { Rota } \\
\text { una vez }\end{array}$ & $\begin{array}{c}\text { Rota } \\
\text { dos veces }\end{array}$ & $\begin{array}{c}\text { Rota } \\
\text { tres veces }\end{array}$ \\
\hline 1. Muy bajo & 50,0 & 23,3 & 16,7 & 10,0 \\
2. Bajo & 47,0 & 15,2 & 15,2 & 22,7 \\
3. Medio & 31,3 & 18,8 & 12,5 & $37,5^{\mathrm{a}}$ \\
4. Alto & 38,5 & 19,2 & 7,7 & $34,6^{\mathrm{a}}$ \\
5. Muy alto & 60,0 & 23,3 & 10,0 & $6,7^{\mathrm{a}}$ \\
Total & 47,0 & 19,0 & 13,1 & 20,8 \\
\hline
\end{tabular}

Fuente: Elaboración propia con datos de la encuesta SADIO-UNGS sobre las competencias tecnológicas de los trabajadores informáticos.

a Prueba Z significativa al $10 \%$.
CUADRO B.5

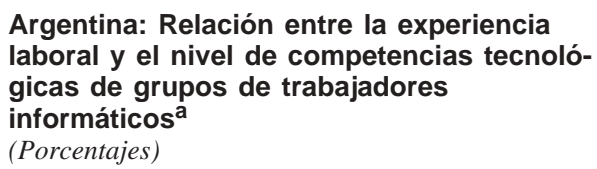

Argentina: Relación entre la experiencia laboral y el nivel de competencias tecnológicas de grupos de trabajadores informáticos ${ }^{\mathrm{a}}$

(Porcentajes)

\begin{tabular}{lcccc}
\hline $\begin{array}{l}\text { Nivel de } \\
\text { competencias } \\
\text { de cada grupo }\end{array}$ & $\begin{array}{c}\text { Años de experiencia } \\
\text { de } 10\end{array}$ & $\begin{array}{c}\text { De } 11 \\
\text { a 20 }\end{array}$ & $\begin{array}{c}\text { De 21 } \\
\text { a 30 }\end{array}$ & $\begin{array}{c}\text { Más } \\
\text { de 30 }\end{array}$ \\
\hline $\begin{array}{llccc}\text { 1. Muy bajo } \\
\text { 2. Bajo }\end{array}$ & 40,0 & 36,7 & 23,3 & 0,0 \\
3. Medio & $62,1^{\text {b }}$ & 27,3 & 9,1 & 1,5 \\
4. Alto & 62,5 & 31,3 & 6,3 & 0,0 \\
5. Muy alto & 61,5 & 23,1 & 7,7 & 7,7 \\
& $16,1^{\text {c }}$ & 48,4 & 22,6 & 12,9 \\
Total & & & & \\
\hline
\end{tabular}

Fuente: Elaboración propia con datos de la encuesta SADIO-UNGS sobre las competencias tecnológicas de los trabajadores informáticos.

a La probabilidad de aceptar la hipótesis nula de ausencia de asociación es $0,02 \%$.

b Prueba Z significativa al $10 \%$.

c Prueba $\mathrm{Z}$ significativa al $1 \%$. 
CUADRO B.6

Argentina: Relación entre la edad y el nivel de competencias tecnológicas de grupos de trabajadores informáticos ${ }^{\mathrm{a}}$

\begin{tabular}{lcccc}
\hline \multirow{2}{*}{$\begin{array}{l}\text { Nivel de } \\
\text { competencias } \\
\text { de cada grupo }\end{array}$} & $\begin{array}{c}\text { De } 21 \\
\text { a } 30\end{array}$ & $\begin{array}{c}\text { De 31 } \\
\text { a } 40\end{array}$ & $\begin{array}{c}\text { De } 41 \\
\text { a } 50\end{array}$ & $\begin{array}{c}\text { De 51 } \\
\text { en adelante }\end{array}$ \\
\hline 1. Muy bajo & 13,3 & 36,7 & 33,3 & $16,7^{\mathrm{b}}$ \\
2. Bajo & $47,0^{\mathrm{c}}$ & 28,8 & 19,7 & 4,5 \\
3. Medio & 50,0 & 31,3 & 12,5 & 6,3 \\
4. Alto & 46,2 & 38,5 & $7,7^{\mathrm{b}}$ & 7,7 \\
5. Muy alto & $9,7^{\mathrm{d}}$ & 51,6 & 29,0 & 9,7 \\
& & & & \\
Total & 34,3 & 36,1 & 21,3 & 8,3 \\
\hline
\end{tabular}

Fuente: Elaboración propia con datos de la encuesta SADIO-UNGS sobre las competencias tecnológicas de los trabajadores informáticos.

a La probabilidad de aceptar la hipótesis nula de ausencia de asociación es $0,07 \%$.

b Prueba $\mathrm{Z}$ significativa al $10 \%$.

c Prueba $Z$ significativa al $1 \%$.

d Prueba $\mathrm{Z}$ significativa al $1 \%$.
CUADRO B.7

\section{Argentina: Relación entre el género y el nivel de competencias de grupos de trabajadores informáticos (Porcentajes)}

\begin{tabular}{lcc}
\hline \multirow{2}{*}{$\begin{array}{l}\text { Nivel de competencias } \\
\text { de los grupos }\end{array}$} & \multicolumn{2}{c}{ Género } \\
\cline { 2 - 3 } Femenino & Masculino \\
\hline 1. Muy bajo & $26,7^{\mathrm{a}}$ & 73,3 \\
2. Bajo & 18,2 & 81,8 \\
3. Medio & 6,2 & 93,8 \\
4. Alto & 15,4 & 84,6 \\
5. Muy alto & 12,9 & 87,1 \\
& & 82,8 \\
\hline
\end{tabular}

Fuente: Elaboración propia con datos de la encuesta SADIO-UNGS sobre las competencias tecnológicas de los trabajadores informáticos.

a Prueba Z significativa al $10 \%$.
Aguirre, J. (2003): La ESLAI: advenimiento, muerte prematura y proyección, Newsletter de Sadio, Buenos Aires, diciembre.

Albornoz, M., L. Luchilo y otros (2003): El talento que se pierde. Aproximaciones al estudio de la inmigración de profesionales, investigadores y tecnólogos argentinos, Documento de trabajo, $\mathrm{N}^{\circ} 4$, Buenos Aires, Centro Redes.

Anlló, G., G. Bezchinsky y otros (2003): El caso de la industria de software y servicios informáticos en Argentina, estudio JICA/ CEPAL sobre fuentes de crecimiento económico en Argentina, Buenos Aires. Disponible en <www.eclac.cl/argentina>

Arora, A. y A. Gambardella (2005): From Underdogs to Tigers. The Rise and Growth of the Software Industry in Brazil, China, India, Ireland and Israel, Londres, Oxford University Press.

Azpiazu, D., E. Basualdo y H. Nochteff (1990): Los límites de las políticas industriales en un período de reestructuración regresiva: el caso de la informática en Argentina, Desarrollo económico, vol. 30, № 118, Buenos Aires, Instituto de Desarrollo Económico y Social (IDES).

Babini, R. (2003): La Argentina y la computadora. Crónica de una frustración, Buenos Aires, Ed. Dunken.

Borillo, J., V. Robert y G. Yoguel (2005): Para pensar la informática en la Argentina: Desafíos a la especialización y a la competitividad, Buenos Aires, Universidad Nacional de General Sarmiento (UNGS)/Editorial Prometeo, en prensa.

Boscherini, F., M. Novick y G. Yoguel (2003): Nuevas tecnologías de información y comunicación. Los límites en la economía del conocimiento, Buenos Aires, Miño.

Brown, J. y P. Duguid (2000): Mysteries of the region: knowledge dynamics in Silicon Valley, en L. Chong-Moon, W. Miller y otros (comps.), The Silicon Valley Edge: An Habitat for Innovation and Entrepreneurship, Stanford, Stanford University Press.

Cabello, R. y R. Moyano (2005): Incorporación de TIC en el sistema educativo. Las restricciones de las competencias endógenas de los educadores, en R. Borello y G. Robert (comps.), Para pen- sar la informática en la Argentina: desafios a la especialización y a la competitividad, Buenos Aires, Editorial Prometeo/ UNGS, en prensa.

Casalet, M. (2003): La conformación de un sistema institucional territorial en dos regiones: Jalisco y Chihuahua vinculadas con la maquila de exportación, en F. Boscherini, M. Novick y G. Yoguel, Nuevas tecnologías de información y comunicación. Los límites en la economía del conocimiento, Buenos Aires, Miño.

Castells, M. (1996): The Rise of the Network Society, vol. 1, Oxford, Blackwell.

Castilla, E., H. Hwang y otros (2000): Social networks in Silicon Valley, en L. Chong-Moon, W. Miller y otros (comps.), The Silicon Valley Edge: An Habitat for Innovation and Entrepreneurship, Stanford, Stanford University Press.

Chudnovsky, D., A. López y S. Melitzko (2001): El sector de software y servicios informáticos en la Argentina. Situación actual y perspectivas de desarrollo, Documento de trabajo, $\mathrm{N}^{\mathrm{o}} 27$, Buenos Aires, Centro de Investigaciones para la Transformación (CENIT).

Cusumano, M. (2000): Making large teams work like small teams: product development at Microsoft, en U. Jürgens (comp.), New Product Development and Production Networks, Berlín, Springer-Verlag.

Dale, J.M. y N. Julien (2003): 'Libre' software: turning fads into institutions?, Research Policy, $\mathrm{N}^{\mathrm{o}} 32$, Amsterdam, Elsevier.

David, P., R. Cowan y D. Forey (2000): The explicit economics of knowledge codification and tacitness, Industrial and Corporate Change, vol. 9, $\mathrm{N}^{\circ}$ 2, Oxford, Oxford University Press.

Dosi, G., A. Pavitt y L, Soete (1990): The Economics of Technical Change and International Trade, Nueva York, New York University Press.

Ducatel, K. (comp.) (1994): Employment and Technical Change in Europe. Work Organization, Skills and Training, Londres, Edward Elgar Publishing Limited. 
Esser, K., W. Hillebrand y otros (1996): Competitividad sistémica: nuevo desafío para las empresas y la política, Revista de la CEPAL, N $\mathrm{N}^{\circ}$ 59, LC/G.1931-P, Santiago de Chile, agosto.

Freeman, Ch., L. Soete y U. Efendioglu (1995): Diffusion and the employment effects of information and communication technology, International Labour Review, vol. 134, No 4-5, Ginebra, Organización Internacional del Trabajo.

Granovetter, M. (1985): Economic action and social structure: the problem of embeddedness, American Journal of Sociology, vol. 91, Chicago, The University of Chicago Press.

Guerrieri, P. (1993): International Competitiveness, Trade Integration and Technological Independence in Major Latin-American Countries, Nápoles, Università degli Studi di Napoli Federico II, inédito.

Henry, N. y S. Pinch (2000): Spatialising knowledge: placing the knowledge community of sport valley, Geoforum, vol. 31, Amsterdam, Elsevier.

Hetzkowitz, H. y L. Leydesdorf (1997): Universities and the Global Knowledge Economies. The Triple Helix of UniversitiesIndustries-Government Relations, Londres, Pinter.

Jacovkis, P. (2003): Breve historia de la computación en la Argentina, disponible en www.sadio.org.ar

Lall, S. (2001): Competitiveness, Technology and Skills, Cheltenham, Reino Unido, Edward Elgar.

Lam, A (2002): Skills and Careers of R\&D Knowledge Workers in the Network Economy, documento preparado para el 13 Congreso Mundial de IIRA (Berlín, 2003), Uxbridge, Middlesex, Londres, School of Business and Management, Brunel University.

López, A. (2003): El sector de software y servicios informáticos en la Argentina ¿es posible una inserción exportadora sostenible?, en F. Boscherini, M. Novick y G. Yoguel, Nuevas tecnologías de información y comunicación. Los límites en la economía del conocimiento, Buenos Aires, Miño.

López-Bassols, V. (2002): ICT Skills and Employment, STI Working Papers, DSTI/DOC(2002)10, París, Organización de Cooperación y Desarrollo Económicos (OCDE).

Lugones, G., C. Bianco y otros (2003): Indicadores de la sociedad del conocimiento e indicadores de innovación. Vinculaciones e implicancias conceptuales y metodológicas, en F. Boscherini, M. Novick y G. Yoguel, Nuevas tecnologías de información y comunicación. Los límites en la economía del conocimiento, Buenos Aires, Miño.

Markusen, A. (2002): Targeting Occupations in Regional and Community Economic Development, Minnesota, University of Minnesota, Humphrey Institute of Public Affairs, inédito.

Martin, S. y S. Rotondo (2005): Redes de proveedores en la industria manufacturera: un análisis desde la difusión de Tics y las competencias endógenas, en R. Borello y G. Robert (comps.),
Para pensar la informática en la Argentina: desafíos a la especialización y a la competitividad, Buenos Aires, Editorial Prometeo/Ungs, en prensa.

Metcalfe, J., R. Ramlogan y E. Uyarra (2002): Economic Development and the Competitive Process, Working Paper, $\mathrm{N}^{\mathrm{o}} 36$, Manchester, University of Manchester, Centre for Research on Innovation and Competition.

Micheli, J. (2003): El trabajo de digitofactura en la economía postindustrial, Espacios globales: espacios del capitalismo, México, D.F., Ed. Carmen Bueno, en prensa.

Monteverde, H. y A. Pérez (2005): Sobre ventanas de oportunidad $\mathrm{y}$ aportes que puede hacer el sistema educativo, en R. Borello y G. Robert (comps.), Para pensar la informática en la Argentina: desafíos a la especialización y a la competitividad, Buenos Aires, Editorial Prometeo/UnGS, en prensa.

National Research Council (1998): Fostering Research on the Economic and Social Impacts of Information Technologies. Report of a Workshop, Washington, D.C., National Academy.

Nemirovsky, A. y G. Yoguel (2001): Dynamics of High-Technology Firms in the Silicon Valley, Working Paper, $\mathrm{N}^{\circ}$ 01-03, Aalborg, Aalborg University, Department of Business Studies. (2004): Renacimiento de Argentina a través de una economía basada en el conocimiento, el rol de los profesionales argentinos en el exterior, Buenos Aires, Instituto de Industria, Universidad Nacional de General Sarmiento, en $<$ www.littec.org >

Novick, M. (2002): La dinámica de oferta y demanda de competencias en un sector basado en el conocimiento en la Argentina, serie Desarrollo productivo, No 119, LC/L.1696-P, Santiago de Chile, CEPAL. Publicación de las Naciones Unidas, $\mathrm{N}^{\circ}$ de venta: S.02.II.G.8.

Perazzo, R., M. Delbue y otros (1999): Oportunidades para la producción y exportación de Software, Documento de trabajo, $N^{\circ} 9$, Buenos Aires, Agencia Nacional de Promoción Científica y Tecnológica.

Reinert, E. (2002): El papel de la tecnología en la creación de ricos y pobres: el subdesarrollo en un sistema schumpeteriano, Cuadernos de difusión, año 7, N 12, Lima, Escuela Superior de Administración de Empresas (ESAN)

Saxenian, A. (2000): The origins and dynamics of production networks in Silicon Valley, en M. Kenney (comp.), Understanding Silicon Valley: The Anatomy of an Entrepreneurial Region, Stanford, Stanford University Press

Suárez, D. (2004): Fuga de cerebros argentina: un fenómeno que continúa. Disponible en <www.littec.org >

Yoguel, G., M. Novick y otros (2004): Información y conocimiento: la difusión de las Tic en la industria manufacturera argentina, Revista de la CEPAL, No 82, LC/G.2220-P, Santiago de Chile, abril. 